\title{
Fecal Microbiota Transplantation: Indications, Methods, Evidence, and Future Directions
}

\author{
Thomas J. Borody • Sudarshan Paramsothy • Gaurav Agrawal
}

Published online: 14 July 2013

(C) The Author(s) 2013. This article is published with open access at Springerlink.com

\begin{abstract}
Fecal microbiota transplantation (FMT) has attracted great interest in recent years, largely due to the global Clostridium difficile infection (CDI) epidemic and major advances in metagenomic sequencing of the gastrointestinal (GI) microbiota, with growing understanding of its structure and function. FMT is now recommended as the most effective therapy for relapsing CDI and, with further refinement, may even be used in "first-time" CDI. There is interest also in other conditions related to GI dysbiosis-for example, inflammatory bowel disease, irritable bowel syndrome, obesity, and diabetes mellitus - although quality evidence is at present lacking. A few trials are now underway in FMT for ulcerative colitis. Many unanswered questions remain, including FMT methodology - for example, optimal route of administration, what makes a "good donor," safety issues, and long-term effects of FMT.
\end{abstract}

Keywords Fecal $\cdot$ Microbiota $\cdot$ Transplantation · Clostridium difficile $\cdot$ Inflammatory bowel disease · IBD . Colitis · Crohn's $\cdot$ Irritable bowel

This article is part of the Topical Collection on Large Intestine

T. J. Borody $(\bowtie) \cdot G$. Agrawal

Centre for Digestive Diseases, Level 1, 229 Great North Road,

Five Dock, Sydney, NSW 2046, Australia

e-mail: thomas.borody@cdd.com.au

G. Agrawal

e-mail: drgagrawal@gmail.com

S. Paramsothy

St Vincent's Hospital Clinical School, Darlinghurst,

NSW 2010, Australia

e-mail: sparam_au@yahoo.com

\section{Introduction}

Fecal microbiota transplantation (FMT) is the introduction of a fecal suspension derived from a healthy donor into the gastrointestinal (GI) tract of a diseased individual. While not a new therapeutic concept $[1,2]$, it has, over the last few years, experienced a significant growth in interest, with an evolving methodology and clinical indications largely due to two factors: (1) the global CDI epidemic and (2) a growing appreciation of the complexity of the GI microbiome and its active role in health and disease. FMT is no longer considered an "alternative," last-resort medical practice but, rather, is now gaining mainstream acceptance as a valuable, although still poorly understood, therapy with biological plausibility. This is reflected in the increasing number of scientific publications related to FMT. In this review, we will summarize the latest evidence, indications, and methods of administering FMT, and provide some insight into future directions and therapeutic potentials.

\section{The Gastrointestinal Microbiota}

The human GI microbiota is considered a tissue, not an organ, and is used in FMT to implant in a recipient's GI tract. To understand the utility of FMT, it is first necessary to appreciate the compositional complexity of the GI microbiota, along with its associated functional implications. There are about $10^{14}$ bacterial cells in our body-10 times more than the roughly $10^{13}$ human cells [3] - and most of these bacterial cells reside in the GI tract. Only about $30 \%$ of the GI microbiota is detectable by culture-based techniques [4]. Detailed sequencing studies suggest that 15,000-36,000 different bacterial species are known to inhabit the human GI tract $[5,6]$, with each individual hosting roughly $500-1,500$ 
species, although only a small subset of these are prevalent. Recent metagenomic sequencing analysis established a human gut microbial gene catalogue, identifying 3.3 million nonredundant microbial genes, approximately 150 times larger than the entire human gene complement [7]. The GI microbiota and their genetic products exist in a complex, but balanced, homeostasis and have important roles in nutrition, energy metabolism, host defense, and immune system development $[8-10,11 \bullet \cdot$. Dysbiosis, or abnormal compositional disturbance of this homeostasis, can be associated with various disease states. Such conditions are potentially amenable to therapy with FMT to correct these changes.

\section{Current Indications}

\section{Clostridium difficile Infection}

FMT is most widely used, and now recommended, in the indication of Clostridium difficile infection (CDI) [12••]. $\mathrm{CDI}$ is an ideal condition for FMT, since it is primarily a GI dysbiosis with Clostridium difficile overgrowth. CDI has traditionally yet counterintuitively been treated with antibiotics such as metronidazole, vancomycin, and, more recently, fidaxomicin or rifaximin. However, antibiotic therapy results in further microbiota damage and in recurrence rates of at least $20 \%$, which rise with each subsequent CDI episode [13-15]. Furthermore, antibiotics do not correct the abnormal microbiome but, rather, potentiate the problem. Conversely, FMT corrects the imbalanced microbiota that underlies CDI pathogenesis by providing the patient with a healthy microbiota that has a structural and functional homeostasis derived from a suitable donor.

Historically, FMT has been offered only in a few specialized centers globally, but in the last few years, there has been growing use of FMT, with numerous publications from many international sites demonstrating efficacy in CDI. Most of these have been uncontrolled, but they have all reported high success rates of around $90 \%$ or more [16-21]. The global experience of FMT in CDI is now in excess of 500 patients.

The first randomized controlled trial involving FMT for relapsing CDI was published earlier this year and demonstrated vast superiority of FMT over traditional antibiotic therapy [22••]. FMT had a success rate of $81 \%$ following a single naso-duodenal infusion and $94 \%$ following a second infusion, while vancomycin $500 \mathrm{mg}$ qid for 2 weeks with or without bowel lavage had only $23 \%-31 \%$ efficacy. After FMT, there was increased bacterial diversity, with increases in Bacteroidetes species, Clostridia class IV and XIVa, and decreased Proteobacteria. No significant adverse events were noted other than mild infusion-related diarrhea and discomfort. The trial was stopped early after interim analysis, given the difference between treatments and success of FMT. This study provided the highest-level evidence for superiority of FMT over current optimal care antibiotics. It also shifted FMT into the focus of mainstream medicine, soon followed by the American College of Gastroenterology formally recommending FMT in its Guidelines for Relapsing CDI (R-CDI) [12••]. Future research in FMT for CDI should now shift to refining the microbiota product, administration methodology, luminal effects of FMT on the resident flora, and assessing safety. An important area to study will be the use of FMT in severe and fulminant CDI and in certain high-risk patient groups, such as the profoundly immunosuppressed.

Another area of FMT use is the treatment of CDI coinfection in inflammatory bowel disease (IBD). The original report of this approach included 6 patients, 4 with UC and 2 with Crohn's disease (CD). All were treated successfully with improvement in colitis symptoms [23]. Other cases of successful treatment have recently been reviewed by Anderson et al. [24•], who identified eight case series/reports with a total of 15 patients treated with FMT for CDI coinfecting IBD (9 UC, $6 \mathrm{CD}$ ). All had resolution of CDI, with $86 \%$ demonstrating improved response to IBD medications. Reddy et al. [25] adds further to our total experience in this area. The coexistence with IBD may require additional infusions to ensure successful CDI eradication [17].

\section{Current Methodology}

FMT transplant material (TM), medically classified as a human tissue, is derived from healthy donors, with no risk factors for transmissible diseases or any issues that may alter the cellular composition, particularly antibiotic use. Published international guidelines by the FMT Working Group outline in detail the FMT donor selection criteria and screening tests [26].

Initially, patients identified their own donors selected from family or friends. Subsequently, some institutions offered the option of an anonymous donor(s). This shifts the burden of donor identification from the patient, creates a pool of tested healthy donors with a track record of cure, and also avoids donors with shared genetic or environmental susceptibilities to the recipient. Donor stool is delivered to the institution within a few hours of passage to undergo (1) dilution, generally with normal saline, (2) homogenization with a blender to achieve a liquid slurry, and then (3) filtration to remove particulate matter to facilitate administration.

Most institutions utilize fresh feces, necessitating collection and processing on the day of planned FMT. Hamilton et al. [17] have adopted an approach of using highly filtered human microbiota mixed with a cryoprotectant and then frozen for storage at $-80{ }^{\circ} \mathrm{C}$ until required for use. This processing removes the fecal smell and reduces the volume of the filtrate. Use of such a standardized, purified tissue has 
been shown to have equivalent clinical efficacy in CDI to that of fresh, partly filtered feces. Furthermore, detailed microbiological studies with 16S rRNA gene sequencing demonstrated stable "engraftment" or "implantation" of donor microbiota with the frozen product [27•], with dramatic shifts in recipient gut microbial communities noted after transplantation.

The route of the TM administration varies considerably between institutions and can be naso-duodenal, transcolonoscopic, or enema based. Some practical issues arise related to naso-duodenal administration. Some patients may not find it "palatable" to have fecal material via the upper gut; the occasional complication, such as vomiting, would be avoided with lower GI infusion, and infusion of lower colonic microbiota into the upper GI tract seems unphysiological and may disturb the local microbial balance. Colonoscopy offers the advantage of allowing direct assessment of the colonic mucosa for disease severity and exclusion of coexistent pathology. Additionally, the use of bowel preparation theoretically may assist in "flushing out" the abnormal host microbiota and facilitate "implantation" of the donor microbiota. Enema administration is effective, cheap, and safe and carries less procedural or institutional admission costs. A systematic review of 325 cases of FMT for CDI suggested a lower success rate for upper gut administration (76\%), as compared with colonoscopy (89 \%) and enema (95\%) administration [28]. A recent analysis had similar findings, with a trend toward higher CDI resolution rates with lower GI, rather than upper GI, administration (91\% vs. $82 \%$ ) [29], although no head-to-head comparison has yet been performed.

\section{Future Indications}

\section{Ulcerative Colitis: Curable in Some}

Increasing evidence supports a microbial influence in the pathogenesis of IBD, likely due to an inappropriate immune response toward a component or components of the microbiota [30, 31]. Evidence for a specific pathogen causing IBD is inconclusive, but there is literature suggesting reduced diversity of luminal microbiota in IBD, with distinct compositional and functional changes. We know that certain bacterial species can be depleted in various disorders. In CDI, reduced Bacteroidetes and Firmicutes have been shown to be replenished after FMT, albeit their activity in clearing CDI is unknown [32]. In IBD, both Bacteroidetes and Firmicutes are deficient [5], and mouse models suggest that such bacteria may play an important role activating regulatory T cells [33-35].

The initial rationale for use of FMT in IBD was that if C. difficile causes colitis with reduced microbial diversity and if FMT can reverse this, a similar treatment could be applied to ulcerative colitis (UC). With this in mind, the lead author treated his first UC patient in early 1988, with others soon following, and FMT treatment in 55 patients with various GI disorders was reported in 1989 [36]. Later, the group detailed longer-term follow-up in six UC cases [37]. Today, some 25 years later, the first patient remains asymptomatic with normal histology. In January 1989, Bennet and Brinkman published a case of non-CDI UC treated successfully using FMT [38]. Bennet stopped his own colitis using large-volume donor enemas 6 months prior. Before FMT, his severe colitis was continuously active for 7 years. At 3 months post-FMT, he was asymptomatic and on no UC therapy for the first time in 11 years. These case reports and others since suggest that $\mathrm{UC}$ is curable in a subgroup of patients.

Anderson et al. [24•] summarized various case reports published on FMT in IBD that had promising findings. Remission was reported in 15 of 24 patients ( $63 \%$ ), cessation of IBD medications in 13/17 (76\%), and reduction of GI symptoms in 19/25 (76 \%). Similarly, Brandt confirmed the activity of FMT in his experience with treating UC $[25,39]$. Recently, the lead author's group reviewed their UC cases treated over the last 24 years [40] and discovered an interesting phenomenon. It seems that although initial FMT may not immediately cure UC, as happens with CDI, over many months to years, the implanted microbiota appears to progressively transform the inflamed UC mucosa to normal, histologically uninflamed mucosa. Twelve of 21 patients (57\%) who had repeat colonoscopy at a mean of 33 months (range 1-198 months) demonstrated complete mucosal healing with normal endoscopic appearance and no histological inflammation (Fig. 1).

A phase 1 trial has just been completed in 10 children and young adults with mild to moderate UC [41]. Patients received 5 days of FMT and were then followed up. At 1-week post-FMT, $33 \%$ achieved clinical remission, and $78 \%$ had a clinical response, with $67 \%$ maintaining response at 4 weeks. This confirms that FMT response in UC/IBD is not as predictable as it is in CDI. There is currently significant interest in this area, with at least four registered trials being conducted assessing FMT for UC in adults (www. clinicaltrials.gov).

\section{Crohn's Disease: FMT a Supporting Role?}

The lead author's experience with treating $C D$ using repeated FMT dates back to 1988 [36], where a 4-month response was observed but poorly sustained. A recent pilot study in 4 refractory patients with $\mathrm{CD}$ was reported, in whom the TM was administered via naso-jejunal tube 3 times over a 2-day period [42]. None had significant clinical or endoscopic improvement at 8-weeks follow-up. Although minor changes in microbial composition were observed (weeks 2 
Fig. 1 A 33-year-old male with ulcerative colitis presented with abdominal pain, bloody diarrhea, and mucus discharge. Failing standard antiinflammatory drugs with frequent relapses, fecal microbiota transplantation (FMT) was introduced. FMT was first administered via a transcolonoscopic route followed by daily enemas, reducing to twice weekly, weekly, and then fortnightly. After 80 FMT infusions, he was passing normal stool once per day and was off all drugs for 7 months. He was recolonoscoped, and the difference is shown

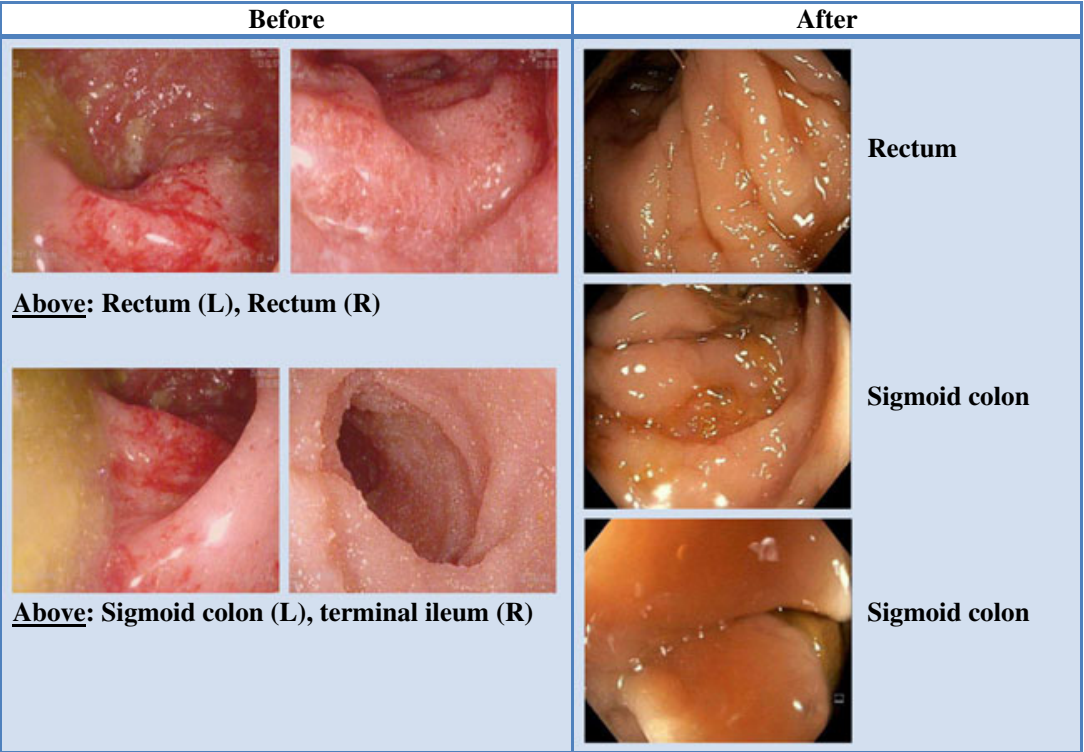

4), by week 8, all patients had returned to their baseline microbial composition, suggesting that unlike in CDI, FMT in these CD patients only transiently implanted at best. FMT appears more appropriate in CD patients with CDI, where a measurable clinical improvement can be gauged and CDI cured (Fig. 2) [23]. Hence, it may be relegated to a supportive role in $\mathrm{CD}$.

\section{Irritable Bowel Syndrome}

Published use of FMT in irritable bowel syndrome (IBS) is limited to some 50 case reports in diarrhea-predominant IBS (D-IBS) [36]. Since that publication, the lead author has treated with FMT, over 5-14 days, more than 300 D-IBS patients whose severe symptoms had failed to respond to conservative measures. Clinical response has been most marked in those with more severe diarrhea and pain, but results in this group are not as dramatic or consistent as in CDI. A few reports on the use of FMT in constipation-predominant IBS are available [43-45]. While anecdotal reports are encouraging, no controlled trials to date have been performed, and these are required to validate efficacy and determine whether the GI microbiota may possess the key for unlocking the etiology and effective treatment of IBS, as in CDI.

\section{Other Conditions}

Other disease states that are closely linked to the GI microbiota, such as obesity [46, 47], metabolic syndrome [48], and diabetes mellitus, may potentially be treated by
Fig. 2 A 14-year-old boy with Clostridium difficile and severe Crohn's ileitis poorly controlled on prednisone and azathioprine was treated with fecal microbiota transplantation, with a total of 60 home infusions. He experienced marked clinical improvement, and, incidentally, his severe facial acne also healed within 7 days. Ileoscopy showed pronounced improvement in the inflammation, and Clostridium difficile infection cure was confirmed upon stool testing

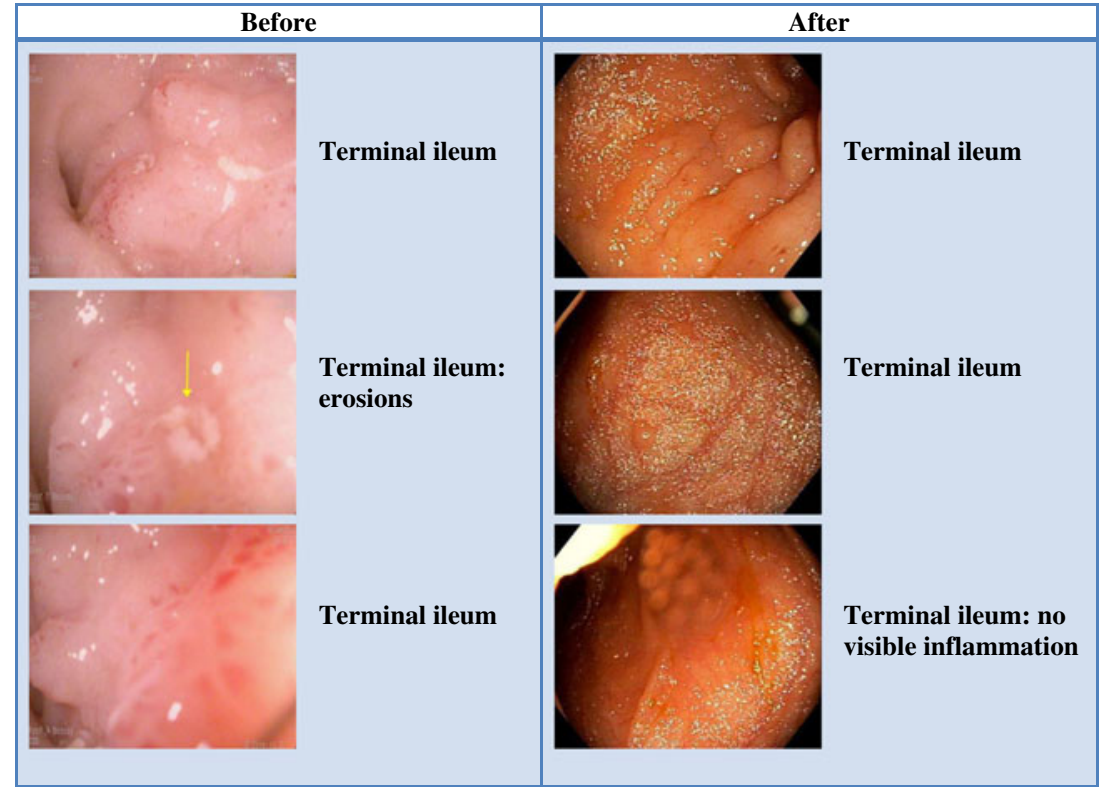


FMT in the future. A recent pilot study assessed the effects of FMT from lean healthy donors to individuals with metabolic syndrome [49]. Six weeks post-FMT, improved insulin sensitivity was noted in the 9 recipients, along with intestinal microbiota changes characterized by increased butyrateproducing bacteria.

Regarding other potential treatable conditions, evidence for FMT at present is lacking. Isolated case reports of FMT response include multiple sclerosis [50], Parkinson's disease [51], chronic fatigue syndrome [52], and idiopathic thrombocytopenic purpura [53]. Apart from these published reports, the lead author has also observed convincing improvement after FMT in several other conditions, including rheumatoid arthritis, sacroileitis, halitosis, acne, insomnia, and major depression. Autism spectrum disorder is another condition in which the GI microbiota is implicated [54], where FMT may have a role.

\section{Unanswered Questions}

Despite the enthusiasm and explosion of research activity in FMT, important questions remain, apart from those addressing potential therapeutic indications. These include whether the TM could be whole flora extract or cultured TM, methods of administration, implantation success, and immunologic responses, as well as the long-term safety implications of altering microbiota composition.

Another crucial question is what makes a "good donor"? The microbiome features of what constitutes a good donor have not been studied using metagenomics and are unknown. Would a "good donor" for CDI, for example, constitute a "good donor" for another indication, or is it a patientspecific problem where the donor needs to be matched to the recipient? Seminal studies have demonstrated that variation of GI microbiota between individuals at a genus and species level tends to congregate within clusters or enterotypes defined by interactions between members of the microbial community [55] and dietary influences [56]. The robustness and predictability of such enterotypes in different cohorts and at phylogenetic and functional levels indicate that they are the result of well-balanced, defined microbial community compositions/host-microbial symbiotic states, of which only a limited number seem to exist across individuals.

Advances in culture-independent molecular microbiological sequencing techniques and powerful bioinformatic programs are allowing increasingly detailed, efficient, and affordable analysis of GI microbiota that will refine our knowledge of its compositional and functional features [57]. Associated with this will be an improved understanding of the impact of FMT on host GI microbiota and the likely therapeutically active/beneficial microbial components, which in turn will allow more meticulous and specific selection of suitable donors. That said, while the GI microbiome and FMT are currently attracting great interest and are very active topical areas of research with enormous therapeutic potential, there is a need to proceed with caution [58].

\section{Future Directions of FMT}

FMT TM began as crude, homogenized human feces, presumably dissolved in water or saline and administered as an enema [2], and similar TM continues to be used currently $[11 \bullet \cdot$. Refinement of this tissue to a highly filtered, frozen colonic microbiota was reported to be equally effective to the crude homogenate by Hamilton et al. [17]. The advantages of this minimally manipulated human tissue TM include equipotency in CDI treatment, absence of fecal smell, quantifiable reproducible cellular content, prolonged viability, and implantation equivalent to the crude homogenate [27•].

Cultured TM, as opposed to a fecal extract, holds some attraction. Cultured "mini" versions of the complete gut microbiota have succeeded in treating CDI in animal studies [59] and a handful of human cases [60, 61]. Lawley et al. [59] designed a mixture of phylogenetically diverse intestinal bacteria that disrupted intestinal dysbiosis, reestablished healthassociated microbiota, and resolved CDI in mice. Tvede et al. [60] in 1989 used 10 cultured human fecal bacteria to cure relapsing CDI in 5 patients. Meanwhile Petrof et al. [61] recently used a culture comprising 33 species of commensal bacteria derived from human feces to successfully treat antibiotic refractory CDI in 2 patients. These are but a few examples of a multitude of potential human microbiota component mixtures that could be developed into "mini" versions of the complete flora and be successful in CDI. Although cultured "mini" versions give the perception of "purity," the fecal smell remains. A further concern is the phenomenon of passaging, by which progressive reculturing alters original bacterial characteristics and function and diminishes the capacity to implant, the paramount problem of probiotics. More important, only in CDI do we have a clue of the compositional deficiencies where such cultured versions may work. In other conditions, such as UC, where apart from reduced diversity the defect is unclear, it makes sense to replace with full-spectrum fecal TM to ensure that any missing components are being replaced. JorupRonstrom et al. [62] reported treating CDI using a mix of human fecal microbes where the entire stool components have been recultivated fortnightly for over 10 years under strict anaerobic conditions, albeit from a single donor. Their success rate of $69 \%$ is less than standard FMT and demonstrates that whole stool culture is possible but that passaging may already be showing loss of efficacy over time in spite of the TM comprising a vast number of subspecies of microbes.

The future TM from all these research efforts may well be the use of lyophilized, encapsulated, and enteric-coated capsules to allow oral administration. Ultimately, it is conceivable 
that after any use of antibiotics, oral FMT will become routine for ecologically conserving our GI microbiome.

\section{Conclusion}

FMT has undergone a rapid transformation in the past decade, from being considered an evidence-free, alternative form of medicine to acceptance as a mainstream treatment option with vast therapeutic potential. While now proven as the most effective therapy for R-CDI, controlled data are lacking with regard to its role in other conditions associated with GI dysbiosis, and high-quality clinical trials are required in these areas. Additionally, further research is required to refine optimal tissue or product composition, as well as mode of FMT administration. Further work will follow in defining the dysbiosis in numerous conditions and in obtaining a greater understanding of the complex microbiological changes after FMT therapy, searching for potential long-term benefits and adverse effects.

Acknowledgment Editor's Note by Brooks Cash: In a letter dated April 25, 2013 to the presidents of the four major U.S.-based Gastroenterology and Hepatology Societies, Karen Midthun, MD, Director of the Center for Biologics Evaluation and Research of the U.S. Food and Drug Administration (FDA), indicated that fecal microbiota transplant is considered both a biological product under the Public Health Service Act [42 U.S.C. 262(i)] and a drug under the Federal Food, Drug, and Cosmetic Act [21 U.S.C. $321(\mathrm{~g})]$. Consequently, use of this technique for therapeutic purposes in the U.S. requires an approved Investigational New Drug Application.

\section{Compliance with Ethics Guidelines}

Conflict of Interest Thomas J. Borody has a pecuniary interest in the Centre for Digestive Diseases, where fecal microbiota transplantation is a treatment option for patients, and he has filed patents in this field.

Sudarshan Paramsothy declares that he has no conflict of interest.

Gaurav Agrawal declares that he has no conflict of interest.

Human and Animal Rights and Informed Consent This article does not contain any studies with human or animal subjects performed by any of the authors.

Open Access This article is distributed under the terms of the Creative Commons Attribution License which permits any use, distribution, and reproduction in any medium, provided the original author(s) and the source are credited.

\section{References}

Papers of particular interest, published recently, have been highlighted as:

- Of importance

-. Of major importance

1. Zhang F, Luo W, Shi Y, et al. Should we standardize the 1,700-yearold fecal microbiota transplantation? Am J Gastroenterol. 2012; 107(11):1755.
2. Eiseman B, Silen W, Bascom GS, Kauver AJ. Fecal enema as an adjunct in the treatment of pseudomembranous enterocolitis. Surgery. 1958;44(5):854-9.

3. Savage DC. Microbial ecology of the gastrointestinal tract. Annu Rev Microbiol. 1977;31:107-33.

4. Eckburg PB, Bik EM, Bernstein CN, et al. Diversity of the human intestinal microbial flora. Science. 2005;308:1635-8.

5. Frank DN, St Amand AL, Feldman RA, et al. Molecular-phylogenetic characterization of microbial community imbalances in human inflammatory bowel diseases. Proc Natl Acad Sci U S A. 2007;104:13780-5.

6. Frank DN, Pace NR. Gastrointestinal microbiology enters the metagenomics era. Curr Opin Gastroenterol. 2008;24(1):4-10.

7. Qin J, Li R, Raed J, et al. A human gut microbial gene catalogue established by metagenomic sequencing. Nature. 2010; 464(7285):59-65.

8. Backhed F, Ley RE, Sonnenburg JL, et al. Host bacterial mutualism in the human intestine. Science. 2005;307:1915-20.

9. Hooper LV, Midtvedt T, Gordon JI. How host-microbial interactions shape the nutrient environment of the mammalian intestine. Annu Rev Nutr. 2002;22:283-307.

10. Garrett WS, Gordon JI, Glimcher LH. Homeostasis and inflammation in the intestine. Cell. 2010;140:859-70.

11. • Brandt LJ. Intestinal Microbiota and the Role of Fecal Microbiota Transplant (FMT) in Treatment of C. difficile Infection. Am J Gastroenterol. 2013;108:177-85. This detailed review of FMT technology and therapeutic applications is quite extensive, with a broad list of references.

12. •- Surawicz CM, Brandt LJ, Binion DG, et al. Guidelines for Diagnosis, Treatment, and Prevention of Clostridium difficile Infections. Am J Gastroenterol. 2013;108:478-98. Latest guidelines for the first time include FMT in the treatment of a subgroup of Clostridium difficile infections.

13. Cohen SH, Gerding DN, Johnson S, et al. Clinical practice guidelines for Clostridium difficile infection in adults: 2010 update by the society for healthcare epidemiology of America (SHEA) and the infectious diseases society of America (IDSA). Infect Control Hosp Epidemiol. 2010;31(5):431-55.

14. McFarland LV. Alternative treatments for Clostridium difficile disease: what really works? J Med Microbiol. 2005;54:101-11.

15. Huebner ES, Surawicz CM. Treatment of recurrent Clostridium difficile diarrhea. Gastroenterol Hepatol. 2006;2:203-8.

16. Brandt LJ, Aroniadis OC, Mellow M, et al. Long-Term follow-up of colonoscopic fecal microbiota transplant for recurrent Clostridium difficile infection. Am J Gastroenterol. 2012;107: 1079-87.

17. Hamilton MJ, Weingarden AR, Sadowsky MJ, et al. Standardized Frozen Preparation for Transplantation of Fecal Microbiota for Recurrent Clostridium difficile Infection. Am J Gastroenterol. 2012;107:761-7.

18. Kelly CR, de Leon L, Jasutkar N. Fecal Microbiota Transplantation for Relapsing Clostridium difficile Infection in 26 Patients Methodology and Results. J Clin Gastroenterol. 2012;46:145-9.

19. Mattila E, Uusitalo-Seppälä R, Wuorela M, et al. Fecal transplantation, through colonoscopy, is effective therapy for recurrent Clostridium difficile infection. Gastroenterology. 2012;142(3): 490-6.

20. Kassam Z, Hundal R, Marshall JK, et al. Fecal Transplant via Retention Enema for Refractory or Recurrent Clostridium difficile Infection. Arch Intern Med. 2012;172(2):191-3.

21. Borody TJ, Khoruts A. Fecal microbiota transplantation and emerging applications. Nat Rev Gastroenterol Hepatol. 2011;9:88-96.

22. ••van Nood E, Vrieze A, Nieuwdorp M, et al. Duodenal infusion of donor feces for recurrent Clostridium difficile. N Engl J Med. 2013;368(5):408. First randomized controlled trial of FMT in treatment of relapsing Clostridium difficile infection. 
23. Borody TJ, Wettstein AR, Leis S, et al. Clostridium difficile complicating inflammatory bowel disease: pre- and post-treatment findings. Gastroenterology. 2008;134:A361-1.

24. - Anderson JL, Edney RJ, Whelan K. Systematic review: fecal microbiota transplantation in the management of inflammatory bowel disease. Aliment Pharmacol Ther. 2012;36:503-16. Broad review of up-to-date information on known FMT treatment in inflammatory bowel diseases.

25. Reddy SS, and Brandt LJ. Clostridium difficile Infection and Inflammatory Bowel Disease. J Clin Gastroenterol 2013 Mar 15 (Epub ahead of print)

26. Bakken JS, Borody T, Brandt LJ, et al. Treating Clostridium difficile with Fecal Microbiota Transplantation. Clin Gastroenterol Hepatol. 2011;9:1044-9.

27. - Hamilton MJ, Weingarden AR, Unno T, et al. High-throughput DNA sequence analysis reveals stable engraftment of gut microbiota following transplantation of previously frozen fecal bacteria. Gut Microbes. 2013;4(2):1-11. This paper describes for the first time the successful implantation of minimally manipulated human gastrointestinal microbiota.

28. Gough E, Shaikh H, Manges AR. Systematic review of microbiota transplantation (fecal bacteriotherapy) for recurrent Clostridium difficile infection. Clin Infect Dis. 2011;53(10):994-1002.

29. Kassam Z, Lee CH, Yuan Y et al. Fecal Microbiota Transplantation for Clostridium difficile Infection: Systematic Review and MetaAnalysis. Am J Gastroenterol 108(4): 500-508

30. Nagalingam NA, Lynch SV. Role of the microbiota in inflammatory bowel diseases. Inflamm Bowel Dis. 2012;18:968-84.

31. Tannock GW. The bowel microbiota and inflammatory bowel diseases. Int J Inflam 2010: 954051.

32. Khoruts A, Dicksved J, Jansson JK, et al. Changes in the composition of the human fecal microbiome after bacteriotherapy for recurrent Clostridium difficile-associated diarrhea. J Clin Gastroenterol. 2010;44:354-60.

33. Round JL, Lee SM, Li J, et al. The Toll-like receptor 2 pathway establishes colonization by a commensal of the human microbiota. Science. 2011;332(6032):974-7.

34. Atarashi K, Tanoue T, Shima T, et al. Induction of Colonic Regulatory T Cells by Indigenous Clostridium Species. Science. 2011;331(6015):337-41.

35. Round JL, Mazmanian SK. Inducible Foxp3+ regulatory T-cell development by a commensal bacterium of the intestinal microbiota. Proc Natl Acad Sci U S A. 2010;107(27):12204-9.

36. Borody TJ, George L, Andrews P, et al. Bowel-flora alteration: a potential cure for inflammatory bowel disease and irritable bowel syndrome? Med J Aust. 1989;150:604.

37. Borody T, Warren E, Leis S, Surace R, Ashman O. Treatment of ulcerative colitis using fecal bacteriotherapy. J Clin Gastroenterol. 2003;37(1):42-7.

38. Bennet JD, Brinkman M. Treatment of ulcerative colitis by implantation of normal colonic flora. Lancet. 1989;1:164.

39. Brandt LJ, Aroniadis OC. Long-term follow-up study of fecal microbiota transplantation (FMT) for ulcerative colitis (UC). Am J Gastroenterol. 2012;107 suppl 1:1626-S657.

40. Borody T, Wettstein A, Campbell J, et al. Fecal Microbiota Transplantation in Ulcerative Colitis: Review of 24 Years Experience. Am J Gastroenterol. 2012;107(S1):S665. Abstract 1644.

41. Kunde S, Pham A, Bonczyk S et al. Safety, Tolerability, and Clinical Response after Fecal Transplantation in Children and Young Adults with Ulcerative Colitis. Journal of Pediatric Gastroenterology \& Nutrition 2013 (in press; online 29/3/2013)
42. Vermeire S, Joossens M, Verbeke K, et al. Pilot study on the safety and efficacy of fecal microbiota transplantation in refractory Crohn's disease. Gastroenterology. 2012;142:S360.

43. Andrews PJ, Barnes P, Borody TJ. Chronic constipation reversed by restoration of the bowel flora.A case and a hypothesis. European J Gastroenterol Hepatol. 1992;4:245-7.

44. Borody $\mathrm{T}$, Leis $\mathrm{S}$, McGrath $\mathrm{K}$ et al. Treatment of Chronic Constipation and Colitis using human probiotic infusions. Probiotics, Prebiotics and New Foods Conference, Universita Urbaniana, Rome, Sept 2-4 2001

45. Borody T, Warren E, Leis S. et al. Bacteriotherapy using fecal flora: Toying with human Motions. J Clin Gastroenterol. Vol 38, No 6, July 2004.

46. Angelakis E, Armougom F, Million M, et al. The relationship between gut microbiota and weight gain in humans. Future Microbiol. 2012;7(1):91-109.

47. Ley RE. Obesity and the human microbiome. Curr Opin Gastroenterol. 2010;26(1):5-11.

48. Zupancic ML, Cantarel BL, Liu Z, et al. Analysis of the Gut Microbiota in the Old Order Amish and Its Relation to the Metabolic Syndrome. PLoS One. 2012;7(8):e43052.

49. Vrieze A, van Nood E, Holleman F, et al. Transfer of intestinal microbiota from lean donors increases insulin sensitivity in individuals with metabolic syndrome. Gastroenterology. 2012;143(4):913-6.

50. Borody TJ, Leis S, Campbell J, et al. Fecal microbiota transplantation (FMT) in multiple sclerosis (MS) [abstract]. Am J Gastroenterol. 2011;106:S352.

51. Anathaswamy A. Fecal transplant eases symptoms of Parkinson's. New Scientist Issue. 2011;2796:19.

52. Borody TJ, Nowak A, Torres M, et al. Bacteriotherapy in chronic fatigue syndrome: a retrospective review[abstract]. Am J Gastroenterol. 2012;107 Suppl 1:S591-592. A1481.

53. Borody TJ, Campbell J, Torres M, et al. Reversal of idiopathic thrombocytopenic purpura (ITP) with fecal microbiota transplantation (FMT) [abstract]. Am J Gastroenterol. 2011;106:S352.

54. Adams JB, Johansen LJ, Powell LD, et al. Gastrointestinal flora and gastrointestinal status in children with autism - comparisons to typical children and correlation with autism severity. BMC Gastroenterol. 2011;11:22.

55. Arumugam M, Raes J, Pelletier E, et al. Enterotypes of the human gut microbiome. Nature. 2011;473(7346):174-80.

56. Wu GD, Chen J, Hoffmann C, et al. Linking long-term dietary patterns with gut microbial enterotypes. Science. 2011;334(6052):105-8.

57. Fraher MH, O'Toole PW, Quigley EM. Techniques used to characterize the gut microbiota: a guide for the clinician. Nat Rev Gastroenterol Hepatol. 2012;9:312-22.

58. 58: Kahn SA, Goeppinger SR, Rubin DT. Fecal Transplantation: a new therapy for IBD? Proceeding with Caution. Inflamm Bowel Dis Monit 2013; accepted manuscript [in press]

59. Lawley TD, Clare S, Walker AW, et al. Targeted Restoration of the Intestinal Microbiota with a Simple, Defined Bacteriotherapy Resolves Relapsing Clostridium difficile Disease in Mice. PLoS Pathog. 2012;8(10):e1002995.

60. Tvede M, Rask-Masden J. Bacteriotherapy for chronic relapsing Clostridium difficile diarrhoea in six patients. Lancet. 1989;1(8648):1156-60.

61. Petrof EO, Gloor GB, Vanner SJ, et al. Stool substitute transplant therapy for the eradication of Clostridium difficile infection: 'rePOOPulating' the gut. Microbiome. 2013;1:3.

62. Jorup-Ronstrom C, Hakanson A, Sandell S, et al. Fecal transplant against relapsing Clostridium difficile-associated diarrhea in 32 patients. Scand J Gastroenterol. 2012;47(5):548-52. 